\title{
Interactive comment on "Tracking the direct impact of rainfall on groundwater at Mt. Fuji by multiple analyses including microbial DNA" by Ayumi Sugiyama et al.
}

\section{Anonymous Referee \#2}

Received and published: 10 May 2016

General comments The data presented in this study is interesting and gives insight into patterns of rainfall influence on groundwater. However, the text, especially the Results and Discussion parts, was hard to follow because of language issues. For example, the results paragraphs describing bacterial community diversity needs editing. The introduction needs changes (please see specific comments below). The Discussion paragraphs mix previous results with results from the actual study, and at times it is hard to figure out which is which. Thus a better organization of the paragraphs is needed.

Specific comments - The introduction needs to be re-written and/or re-arranged. Some paragraphs seem like a discussion, and the relevant principal information is difficult

Printer-friendly version

Discussion paper 
to extract. Also it is difficult to understand what the last 2 sentences (P3-L4-L7) the introduction mean or want to show, and should probably be inserted before in the introduction. - Material and Methods P3-L23: How many samples were taken? Were different time points used? Was only one groundwater sample from one site (G1?) taken for microbial diversity analysis? P4-L2: Please explain and rephrase 'Rainwater samplers prevented form evaporation' - Results P5-L23: It is unclear to me whether the rainfall and groundwater samples for one event were taken at the same date or with an interval of a few hours or days? A delay in sample recovery from one to the other could be necessary in order to observe an influence of rainfall on the groundwater? P7-L1: Again it might nice to have a table in the supplementary material with all the rainfall and groundwater samples that were taken, and at each date, and for what types of analyses (chemical or microbial). It is again difficult to understand which samples were used for microbial analyses compared to those used for the chemical analyses. P7-L16L17: Please rephrase this sentence. P7-L15: Is there a specific reason why the authors chose to discuss the bacterial (and archaeal) community diversity at the order level? P7-L28: The authors should manually check their archaeal taxonomy affiliation. In my experience the sequences affiliated with the Halobacteriales and the Parvarcheota with automated databases can give inaccurate results. It might be worth BLASTing a few OTU representative sequences to make sure their affiliations are correct. P8-L5: 'unique', are the authors describing their results and saying that the archaea in this specific sample are unique? Or that compared to other studies they are unique? Discussion P8-L9: 'shortly', how much time exactly? P8-L30: by 'extraction' do the authors mean 'infiltration'? P9-L3: Do the authors mean DNA-based sequences affiliated with thermophilic bacteria? P9-L5: 'viral particles were used', is this study or in a previous study? P9-L15: 'clones', were clone libraries constructed as well? P9-L20: 'absolute', do the authors mean 'strict anaerobes'?

Printer-friendly version

Discussion paper

Interactive comment on Biogeosciences Discuss., doi:10.5194/bg-2016-78, 2016.

Interactive

comment 